\title{
EL: the new aromaticity measure based on one-electron density function
}

\author{
Justyna Dominikowska • Marcin Palusiak
}

Received: 6 December 2011 / Accepted: 20 December 2011/Published online: 18 January 2012

(C) The Author(s) 2012. This article is published with open access at Springerlink.com

\begin{abstract}
Ellipticity of the bond, being the quantity which numerically reflects how far the given chemical bond has elliptic cross-section, may be used to estimate $\pi$-electron contribution in bonding. We make use of that fact and develop a new measure of aromaticity-EL index. Since ellipticity is available from calculations on oneelectron density function, EL can be used for both theoretical and experimental data. The EL measure is normalized to make interpretation of this parameter as easy and comfortable as possible. We compare EL values with the values of other commonly used aromaticity measures, such as HOMA, PDI, FLU, and NICS. It appears that the indications of EL are in agreement with indications of other indices and general expectations.
\end{abstract}

Keywords Aromaticity · Aromaticity index · Bond ellipticity · Bond critical point · QTAIM

\section{Introduction}

Although it is one of the most important concepts in chemistry, aromaticity still has no unified definition [1-3]. It was postulated in many reports [4-14] and summarized in Tetrahedron report 520 [3] that aromaticity is a collective phenomenon and hence a variety of criteria should be used in the discussion of this term in any particular subject of investigation [15]. For this

Electronic supplementary material The online version of this article (doi:10.1007/s11224-011-9941-6) contains supplementary material, which is available to authorized users.

J. Dominikowska $\cdot$ M. Palusiak $(\bowtie)$

Department of Theoretical and Structural Chemistry, University

of Łódź, Pomorska 163/165, 90-236 Łódź, Poland

e-mail: marcinp@uni.lodz.pl reason several measures of aromaticity were introduced on the basis of unique physical and chemical properties of the species considered to be aromatic. In general, aromaticity is directly associated with the specific $\pi$-electronic structure resulting from the delocalization of $\pi$-electrons along the sequence of bonds forming the aromatic ring. This particular property of aromatic systems is responsible for unique geometrical, magnetic, and electron properties which in turn are reflected in chemical behavior of aromatic species. There are different aromaticity indices which were thoroughly used to estimate the aromatic character. Let us mention here the most successful ones such as HOMA [16, 17]-based on molecular geometry, NICS [18, 19]—based on induced magnetic ring currents, and finally those based on the properties of electron density function, e.g., PDI ( $\pi$-electron delocalization index) [20], FLU (fluctuation index) [15] or MCI (multi-center index) [21, 22], BOIA (bond order index of aromaticity) [22], and $\theta$ index [23]. It is remarkable that all aromaticity measures defined on the basis of properties of electron density function are based on the number of electrons shared by two or more atomic centres (or atomic basins in accordance with the quantum theory of atoms in molecules (QTAIM) [24]). In other words, those aromaticity measures are constructed on di- or multi-center indices. Because of this the one-electron density function (e.g., this obtained in experimental charge density measurements) is not a sufficient source of information for the estimation of the above-mentioned electron density-based aromaticity indices.

In this article, we introduce a new measure of aromaticity based on hessian eigenvalues obtained from oneelectron density function ${ }^{1}$. This new and easy to use index

\footnotetext{
${ }^{1}$ We define the term one-electron density as the probability of finding the single electron in the given point of space, no matter which one of the electrons of the molecular system it is.
} 
of aromaticity can be applied for both theoretical and experimental electron density data.

\section{Methodology}

To compare selected QTAIM-based parameters of different $\mathrm{CC}$ bonds we selected a few systems, namely: benzene, 1,3-cyclohexadiene, 1,4-cyclohexadiene, cyclohexene, 1,3butadiene (in its three conformations: s-trans, gauche-cis and $s$-cis), (3E)-hexa-1,3,5-triene, propene, ethylene, and ethane. The geometries of these systems were optimized using DFT [25, 26] methods implemented in Gaussian09 [27] program. The B3LYP [28-31] functional was applied together with the $6-311++\mathrm{G}(\mathrm{d}, \mathrm{p})$ [32] basis set. The optimization procedure was performed using very tight optimization criteria (Opt $=$ VeryTight in Gaussian09) and large DFT integration grid (Int = Ultrafine in Gaussian09). For optimized geometries the analysis of electron distribution function was performed according to Bader's atoms in molecules theory [24, 33]. The analysis was carried out using AIMAll program [34].

The following systems were selected for the proper part of the study: benzene, naphthalene, anthracene, phenanthrene, triphenylene, cyclobutadiene, benzocyclobutadiene, biphenylene, azulene, cyclopent[f,g]acenaphthylene, pyrene, fulvene, cycloocta-1,3,5,7-tetraene, and tetrakis(cyclobutadieno)cyclooctatetraene (Fig. 1). All the systems were optimized in the same way as the ones described in the previous paragraph. The frequency analysis on the same level of theory (B3LYP/6-311++G(d,p)) was carried out in order to verify if the optimized geometries correspond to stationary points. No imaginary frequencies were found.

For the description of aromaticity of those systems various aromaticity indices were used. The indices applied in this study were: HOMA [16, 17]—geometry-based indicator of aromaticity with its components $[35,36] \mathrm{EN}$ and GEO, NICS [18] — founded on magnetic properties of systems, PDI [20] and FLU [15] - both based on electron delocalization indices [37, 38]. Definitions of the abovementioned indices can be found in the ESI associated with this article.

\section{Results}

The ellipticity of the bond

QTAIM gives the unique possibility to have an insight into a region of a system on the basis of physical properties of that system, i.e., it gives the possibility to divide the system (e.g., a molecule) into subsystems (e.g., atoms) on the basis of zero-flux in the electron density gradient field. Since the majority of chemists are interested in the relationship between the properties of the molecular fragment (e.g., reaction center, substituent group, chemical group of some particular importance, etc.) and chemical properties of the given chemical species (such as reactivity, ability of formation of intermolecular interactions, some specific structural properties, etc.), QTAIM has become one of the most powerful utilities of modern chemistry, forming a bridge between advanced theoretical, and experimental techniques. In particular the properties of the electron density function in the so-called bond critical point (BCP, the $(3,-1)$ saddle point on electron density curvature being a minimum in the direction of the atomic interaction line and a maximum in two directions perpendicular to it) seem to be valuable information for chemists, since it was proven in many papers that the chemical bonding can be characterized and classified on the basis of electron density characteristics measured in BCPs [24, 39-42]. It was emphasized in many papers dealing with QTAIM applications that the presence of a bond path (BP, the line linking points of maximum electron density along the direction of the bond) linking a pair of atoms and the BCP corresponding to it fulfills the sufficient and necessary condition requiring that the atoms be bonded to one another $[43,44]$. The above statement can be used as the universal criterion for the presence of the chemical bond, no matter what kind of bond it is. It was also demonstrated that the strength of the given chemical bond is related to the amount of electron density in $\operatorname{BCP}\left(\rho_{\mathrm{BCP}}\right)$. This observation was made for both shared bonds [45-50] and the bonds of closed-shell character [51-55] (Note that some exceptions were also reported [56-58]). There are also other parameters of electron density function in $\mathrm{BCP}$, which may give valuable information on the bonding, such as the laplacian of electron density, $\nabla^{2} \rho \mathrm{BCP}$, and its components being the eigenvalues of hessian in $\mathrm{BCP}^{2}$.

In this article, we make use of the bonding properties reflected in the curvature of electron density (more precisely the one-electron density). According to QTAIM for the given point in space it can be defined the eigenspace of that point in which three eigenvectors run along directions of maximum electron density changes. Thus, there are three eigenvalues (usually denoted as $\lambda_{1}, \lambda_{2}$ and $\lambda_{3}$ ), each of them corresponding to the proper eigenvector. The larger is the given eigenvalue, the faster changes the electron

\footnotetext{
${ }^{2}$ In this article, we do not discuss the full electron density topology resulting from the properties of the gradient vector field. There are excellent reviews on that topic available in the literature, for instance refs. 21, 37, and 38. Here, we focus our attention only on the parameters which are directly connected with the undertaken issue. We also omit the discussion on atomic properties available from the basins integration, since in our approach we use those parameters only which are available directly from the one-electron density function.
} 
density in the direction corresponding to the proper eigenvector. The sign of the eigenvalue is related to the character of electron density change, being negative when electron density decreases in the related direction, and positive, when electron density increases in such a direction (in respect to $\mathrm{BCP}$ position). In the case of $\mathrm{BCP}$, which is the minimum of electron density in direction along BP and maximum in directions perpendicular to $\mathrm{BP}$, one of the eigenvalues is always positive, whereas two of them are always negative.

The laplacian of electron density, $\nabla^{2} \rho$, is just the sum of hessian eigenvalues, but if it is estimated in BCP, it may give valuable information about the bonding. It was demonstrated for instance that in the case of covalent bonds $\nabla^{2} \rho$ is negative, whereas for chemical bonds of closedshell character it adopts positive values. This results from specific electron density distribution in molecules. If two atoms are connected via ionic bond, the electron density in BCP corresponding to the bond linking these atoms is relatively small (thus, the eigenvalues corresponding to eigenvectors running in directions perpendicular to the BP are also relatively small), but it grows rapidly in direction along the BP (which is reflected by a large value of the eigenvalue corresponding to the eigenvector running in direction of BP). In the case of covalent bonds the amount of electrons in BCP is relatively large, thus the intensity of changes in directions perpendicular to $\mathrm{BP}$ predominates over the intensity of changes along BP. Therefore, we can see that local properties of electron density may reflect crucial, from the chemist's point of view, properties of the chemical molecule.

Let us now have a look on how the electron density may change in directions perpendicular to the bond. Obviously there are two possibilities; the changes may proceed with the same intensity along both eigenvectors perpendicular to $\mathrm{BP}$, or in the case of one of the directions the changes will be more extensive. Therefore, in the former case we have $\lambda_{1}=\lambda_{2}$, whereas in the latter case $\lambda_{1}<\lambda_{2}$ (Note that in BCP both $\lambda_{1}$ and $\lambda_{2}$ are negative by definition, therefore, $\left.\left|\lambda_{1}\right|>\left|\lambda_{2}\right|\right)$. The situation with $\lambda_{1}=\lambda_{2}$ corresponds to the cylindrical shape of the bond cross-section, whereas $\lambda_{1}<\lambda_{2}$ corresponds to the elliptical shape of the bond cross-section. The shape of the cross-section is directly related to the physical properties of the chemical bond. The typical single covalent bond has a cylindrical cross-section. When passing from the single to double bond, the crosssection becomes more and more elliptic, adopting the maximum of ellipticity for a typical double bond. Then, when the bond order increases to that typical of the triple bond, the cross-section becomes cylindrical again (Note that in this article we consider bonds being of bond order in between formally single and formally double). Therefore, it can be said that the case when $\lambda_{1}=\lambda_{2}$ can be characteristic of $\sigma$-type (formally single) bonds, whereas $\lambda_{1}<\lambda_{2}$ corresponds to the cross-section of the bond of at least partially double character. On this basis the parameter known as ellipticity of the bond $(\varepsilon)$ was defined as follows:

$\varepsilon=\frac{\lambda_{1}}{\lambda_{2}}-1$

It was demonstrated that in the case of bonds between two carbon atoms $\varepsilon$ correlates linearly with such bond parameters as bond length or two-center delocalization index [23]. The latter can be interpreted as QTAIM-based bond order, assuming that the two atoms formally form the chemical bond. Therefore, earlier studies show that the values of $\varepsilon$ may numerically reflect $\pi$-electron contribution in bonding. Let us have a look on some examples important in the light of our studies. Table 1 collects bond lengths and ellipticities of CC bonds in selected simple molecules.

As it can be seen, all $\varepsilon$ values are in the range between 0.0 and 0.366. The former value was obtained for the CC bond in ethane, whereas the latter was obtained for the formally double bond in cyclohexa-1,4-diene. For the collection of data in Table 1 the general relation between $\mathrm{CC}$ bond length and ellipticity is very close to linear, with the correlation coefficient of -0.991 . Remarkably, the shortest bond is that in ethene, however, there are bonds which are slightly more elliptic than that in ethene. For instance, the bonds in propene, cyclohexene and cyclohexa-1,4-diene are more elliptic. This means that in the case of those bonds the bond cross-section is more elliptic than in ethene. Why? The only explanation seems to be that in the case of those bonds the ratio of $\pi$ - and $\sigma$-contribution is larger than in ethene. There must be conditions which lead to the weakening of $\sigma$-bonding or the strengthening of $\pi$-bonding in these molecular systems (with respect to ethene). This can be the effect of hyperconjugation with the formally single $\mathrm{CC}$ bond, since in propene some contribution of $\pi$-electrons in the formally single bond can be observed. In the case of butadiene in two conformations the $\pi$-contribution in the formally single bond is remarkable, being larger in the trans conformer. This is connected with the fact that in the gauche-cis conformer steric effects lead to non-planar arrangement of $\mathrm{CC}$ bonds, which lowers the possibility of $\pi$-type conjugation between two double bonds (see SI file for atomic coordinates). In (3E)-hexa-1,3,5-triene (zig zag-like conformation) the lateral double bonds are more elliptic than their inner counterpart, which results from the fact, that the inner double bond is involved in $\pi$-type conjugation in a more effective way than lateral double bonds. All these observations show how sensitive parameter the ellipticity can be. Even small changes in bond lengths may result in big differences in bond ellipticities. Since, as it was shown, ellipticity is the parameter which measures the contribution 
Table 1 Bond lengths (in $\AA$ ) and ellipticities of the selected CC bonds discussed in the text

\begin{tabular}{llll}
\hline Molecule & $\begin{array}{l}\text { C atoms forming } \\
\text { the bond }\end{array}$ & $d(\mathrm{C}, \mathrm{C})$ & $\varepsilon$ \\
\hline Ethane & 1,2 & 1.531 & 0.000 \\
Ethene & 1,2 & 1.329 & 0.330 \\
Benzene & $1,2(2,3 ; 3,4 ; 4,5 ; 5,6)$ & 1.395 & 0.199 \\
Cyclohexa-1,3-diene & 5,6 & 1.537 & 0.004 \\
& $4,5(6,1)$ & 1.510 & 0.039 \\
& 2,3 & 1.466 & 0.096 \\
& $1,2(3,4)$ & 1.341 & 0.329 \\
Cyclohexa-1,4-diene & $2,3(3,4 ; 5,6 ; 6,1)$ & 1.505 & 0.041 \\
& $1,2(4,5)$ & 1.332 & 0.366 \\
Cyclohexene & 4,5 & 1.534 & 0.008 \\
& $3,4(5,6)$ & 1.536 & 0.006 \\
s-trans-buta-1,3-diene & $2,3(6,1)$ & 1.508 & 0.041 \\
& 1,2 & 1.335 & 0.362 \\
(3E)- hexa-1,3,5-triene & $2,3(4,5)$ & 1.456 & 0.088 \\
& $1,2(3,4)$ & 1.338 & 0.318 \\
Propene & $1,2(5,6)$ & 1.449 & 0.101 \\
& 3,4 & 1.341 & 0.313 \\
Gauche-cis-buta-1,3-diene & 2,3 & 1.350 & 0.299 \\
& $1,2(3,4)$ & 1.469 & 0.075 \\
& 2,3 & 1.336 & 0.327 \\
& $1,2(3,4)$ & 1.471 & 0.083 \\
& 2,3 & 1.337 & 0.319 \\
& 1,2 & 1.500 & 0.033 \\
& 2,331 & 0.350 \\
\hline
\end{tabular}

Carbon atoms numbering corresponds to standard numeration being in agreement with chemical nomenclature. Note that $s$-cis-buta-1,3diene is a planar transition state of gauche-cis-buta-1,3-diene

from $\pi$-type bonding, we decided to use it as a measure of aromaticity. In the next section we introduce aromaticity index based on ellipticity of the bond.

EL: a new measure of aromaticity

Probably the most widely used index of aromaticity is the HOMA index (Harmonic oscillator model of aromaticity). There are probably two reasons of that fact. First, HOMA is based on bond lengths, parameters being the ground-state properties which are easily available from both experimental and theoretical techniques. Second, HOMA is very clear in interpretation. As normalized it adopts specific limiting values, that is, 1.0 for fully aromatic system (i.e., benzene) and 0.0 value for the nonaromatic system. Negative values of HOMA are characteristic of antiaromatic systems.

HOMA index can be defined by the following expression $[16,17]$ :
$\mathrm{HOMA}=1-\frac{1}{n} \sum_{j=1}^{n} \alpha_{i}\left(R_{\mathrm{opt}, i}-R_{j}\right)^{2}$

where $n$ represents the number of bonds forming the ring, $\alpha_{i}$ is normalization constant chosen to give HOMA $=0$ for Kekule benzene structure (with the CC bond length as in 1,3-butadiene) and 1 for all the systems with all the bonds equal to the optimal value $R_{\text {opt }}$. HOMA value is close to 1 for aromatic systems, close to 0 for nonaromatic ones and negative for antiaromatic ones.

Since good ideas should be followed, we decided to construct our new measure of aromaticity in a similar way as the HOMA was defined, such that it will be a normalized parameter as easy in interpretation as possible. The second important issue is that the source data of our aromaticity index should also be easily available, being simultaneously the ground-state property which reflects $\pi$-electron contribution to the bonding. The ellipticity of the bond is an obvious choice here. As it was shown in the previous subsection, it is a very sensitive parameter which directly reflects the contribution from $\pi$-type bonding. Importantly, it is also an easily available parameter which can be obtained from one-electron density. The latter can be derived both from quantum chemistry as well as from the experimental charge density measurement. It should be noted that ellipticity is a second-order parameter defined on the basis of hessian eigenvalues estimated in BCP. Thus, our measure of aromaticity could be defined directly from $\lambda_{1}$ and $\lambda_{2}$. However, ellipticity is much easier in interpretation than $\lambda_{1}$ and $\lambda_{2}$ and-which is also very importantsome advanced programs dedicated to QTAIM analysis, such as AIMAll program [34] by Todd A. Keith, give ellipticity values in the standard output.

Since our aim is to define a normalized parameter, it is necessary to define in the first step the reference systems. We need two types of reference systems-one being a fully aromatic system and other that could be considered as fully nonaromatic one. It is very easy to find the fully aromatic system, since in this case the benzene, which is the archetype of the aromatic molecule, is the obvious choice. However, the choice of the nonaromatic reference is more complicated. Probably the best would be the localized benzene counterpart, that is, the hypothetic molecule of cyclohexa-1,3,5-triene. Although it would be possible to obtain such a structure forcing the localization of $\pi$-electrons by the use of advanced quantum-chemical approaches, yet in our opinion this is not a good idea, since it would require referring to a purely virtual molecule. Therefore, we think that the bond lengths in a real molecular system would make better reference numbers. For instance, the bond lengths in butadiene might be appropriate to use, since in the butadiene molecule there 
<smiles>c1ccccc1</smiles>

1.<smiles>c1ccc2ccccc2c1</smiles>

2.<smiles>c1ccc2cc3ccccc3cc2c1</smiles>

3.<smiles>c1ccc2c(c1)ccc1ccccc12</smiles><smiles>C1=CC=C1</smiles>

6.

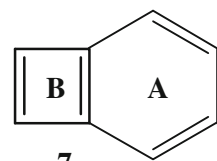

7.

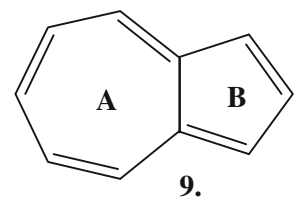<smiles>C1=Cc2ccc3c4c(ccc1c24)C=C3</smiles>

10.<smiles>c1cc2ccc3cccc4ccc(c1)c2c34</smiles>

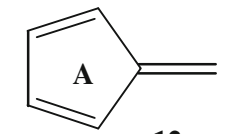

12.

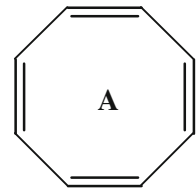

13.

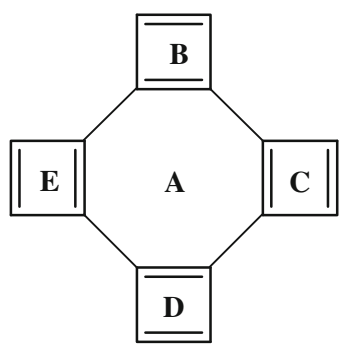

14.
Fig. 1 Schemes of molecular systems under discussion. Ring notation refers to data in Table 2 are formally double and single bonds which may be conjugated via $\pi$-type orbitals, but no cyclic aromatic delocalization can take place in that case. However, in our opinion the case of (3E)-hexa-1,3,5-triene is the best choice. There are several reasons speaking in favor of this compound: (1) it consists of six $s p^{2}$ hybridized carbon atoms, similarly as the hypothetical 1,3,5-cyclohexatriene, (2) it is planar, so there are no steric effects which would affect the $\pi$-electron structure of this system, (3) there is a possibility of $\pi$-type conjugation, like in butadiene and 1,3,5-cyclohexatriene, but no cyclic aromatic delocalization can occur, and finally (4) the surrounding of the inner double bond in 1,3,5-hexatriene is topologically most similar to that in hypothetical 1,3,5-cyclohexatriene. Therefore, we consider such hypothetical molecule of cyclohexa-1,3,5-triene with double and single bond lengths corresponding to the lengths of the inner double bond and the single bond in (3E)-hexa-1,3,5-triene as the reference nonaromatic system.

The main idea of our measure of aromaticity is the same as that which inspired the authors of HOMA index, namely, we want to have a parameter which will show how far a given system is similar to the aromatic archetype. The criterion of this similarity must reflect the character of the bonding, first of all the $\pi$-electron distribution. Since, as it was shown in previous sections, ellipticity of the bond is a parameter which may be used for our purposes, we may define our new index as follows:

$e l_{1}=\sum_{i=1}^{n}\left|\varepsilon_{i}-\varepsilon_{\mathrm{ref}}\right|$

When in the above equation $\varepsilon_{\text {ref }}$ is equal to the ellipticity of the bond in benzene, and the summation runs over all $n$-bonds of CC type in the system, we will have the parameter which allows us to estimate how far the given system is similar to benzene. $e l_{1}=0$ would mean that this similarity is maximal. However, the above equation in its present form would work in accordance with our expectations only for systems consisting of six bonds. For a larger number of bonds the sum will be greater by definition, even for the set of bonds of ellipticity relatively similar to that in benzene. Thus, it is necessary to weight the value of the aromaticity measure with respect to the single bond. In addition, it would be worth normalizing the measure of aromaticity to facilitate its interpretation. Therefore, we introduce the modification of Eq. 3 in the following way:

$e l_{2}=\frac{c}{n} \sum_{i=1}^{n}\left|\varepsilon_{i}-\varepsilon_{\mathrm{ref}}\right|$

where $n$ is still the number of bonds, whereas $c$ is the normalization constant estimated in the same way as $\alpha$ constant in HOMA definition, such that $e l_{2}$ adopts a value 
of 1.0 for the system assumed to be the nonaromatic counterpart of benzene. According to our earlier discussion we consider hypothetical molecule of cyclohexa-1,3,5triene with double and single bond lengths corresponding to the lengths of the inner double bond and the single bond in (3E)-hexa-1,3,5-triene as the reference system. So now $e l_{2}$ adopts a value of 0.0 for the system considered as fully aromatic and 1.0 for the system considered as fully nonaromatic. These are exactly opposite values in comparison with those obtained by HOMA. HOMA is scaled in more natural-let us say-intuitive way. For this reason we follow the inventors of HOMA index and finally define EL index according to Eq. 5:

$\mathrm{EL}=1-\frac{c}{n} \sum_{i=1}^{n}\left|\varepsilon_{i}-\varepsilon_{\mathrm{ref}}\right|$.

Although EL is defined in a very similar way to HOMA index, yet there is one clear difference (of course except the fact, that EL is based on one-electron density properties instead of bond lengths): in HOMA index the $R_{\mathrm{opt}, i}-R_{j}$ term is additionally squared, probably due to the fact that for significantly aromatic systems the difference in bonds lengths with respect to reference bonds in benzene is very small. Since ellipticity is a very sensitive parameter, it is not necessary to amplify the differences in ellipticities for significantly aromatic systems, thus we introduce the modulus instead of the squared value. In the next section of our paper we test the EL index against the set of molecules of different character of aromaticity. As it will be shown, the modulus instead of the squared value gives EL scaled in the range between 1 and -1 for the whole set of systems, including those most aromatic and most antiaromatic. In addition, we compare the EL values with values of other commonly used aromaticity indices, including HOMA, NICS, PDI, and FLU.

\section{EL in use}

Table 2 collects the values of several aromaticity indices estimated for molecular systems shown in Fig. 1. When choosing the systems for further analysis we wanted to have possibly large spectrum of aromaticity. Therefore, we investigated polycyclic aromatic hydrocarbons, in which all possible topological situations from the point of view of Clar's concept of aromatic sextet were taken into account (systems 1-5), molecules containing cyclobutadiene and cyclooctatetraene, being the archetype of antiaromatic and nonaromatic rings, respectively (systems 6-8, 13, 14), azulene and fulvene being the valence isomers of naphthalene and benzene, respectively, and some other exemplary systems which complement the set of rings which are interesting for our analysis. Similarly as HOMA, also EL can be used not only for the given ring but also for any sequence of bonds for which the degree of $\pi$-delocalization is worth considering. Therefore, in the case of systems 2 and 9 we additionally estimate global aromaticity using HOMA and EL measured along all peripheral bonds.

As can be seen in Table 2, in general the indications of different aromaticity indices are in agreement with each other. This concerns also EL. For instance it nicely predicts local aromaticity of the rings in systems 1-5. These predictions are in agreement with Clar's concept of aromatic sextet [59]. Therefore, the most aromatic is the benzene ring (1) (the isolated Clar's sextet), less aromatic are the rings in naphthalene (2) and anthracene (3) (these are the rings with so-called migrating sextet), lateral rings in phenanthrene (4) are clearly more aromatic with respect to their inner counterpart, whereas in triphenylene (5) lateral rings are twice as aromatic as the corresponding inner so-called empty ring. In the case of cyclobutadiene (6) and its derivatives $(\mathbf{7}, \mathbf{8})$ EL adopts a negative value, which corresponds to antiaromatic character of the four-membered rings. It also properly predicts the lower aromaticity (with respect to benzene) of six-membered rings in $\mathbf{7}$ and $\mathbf{8}$. Unlike in the other aromaticity indices, in the case of azulene (9) EL predicts the more aromatic five- and sevenmembered rings with respect to naphthalene. Also in the case of five-membered rings in $\mathbf{1 4}$ the EL shows rather nonaromatic character of the proper rings, whereas HOMA and NICS values suggest antiaromatic character of these rings. This might suggest that EL overestimates the degree of delocalization in this kind of systems. However, the fulvene ring is predicted to be antiaromatic, as expected. Also in the case of cycloocta-1,3,5,7-tetraene EL adopts a negative value, which is in agreement with indications of the other indices and our expectations. It is worth mentioning, that for the whole set of systems investigated (including benzene - the archetype of aromatic system, and cyclobutadiene - the archetype of antiaromatic system) EL gives values in the range from -1 to 1 . This allows very convenient and easy in interpretation analysis with the use of EL measure.

If we consider the global aromaticity of naphthalene and azulene, a comparison can be made only for EL and HOMA, since from the selected set of aromaticity measures only both these indices can be estimated for the sequence of bonds for which $\pi$-electron delocalization can be considered. For naphthalene HOMA and EL are equal to 0.837 and 0.618 , respectively, whereas for azulene these values are 0.966 and 0.876 , respectively. Thus, some difference can be noticed for the former system, whereas for the latter system indications of HOMA and EL are practically the same.

Usually general interdependencies between the values of aromaticity indices are discussed when the larger set of 
Table 2 The values of various aromaticity indices estimated for individual rings in the molecular systems selected for analysis

\begin{tabular}{|c|c|c|c|c|c|c|c|c|c|c|}
\hline System & Ring & HOMA & EN & GEO & PDI & FLU & NICS & $\operatorname{NICS}(1)$ & $\operatorname{NICS}(1)_{\mathrm{zz}}$ & EL \\
\hline 1 & A & 0.989 & 0.011 & 0.000 & 0.103 & 0.000 & -8.06 & -10.23 & -29.25 & 1.000 \\
\hline \multirow[t]{2}{*}{2} & A & 0.783 & 0.082 & 0.134 & 0.075 & 0.009 & -8.39 & -10.48 & -29.09 & 0.591 \\
\hline & B & 0.783 & 0.082 & 0.134 & 0.075 & 0.009 & -8.39 & -10.47 & -29.03 & 0.591 \\
\hline \multirow[t]{3}{*}{3} & A & 0.629 & 0.127 & 0.244 & 0.065 & 0.015 & -7.28 & -9.44 & -26.00 & 0.433 \\
\hline & B & 0.720 & 0.168 & 0.112 & 0.065 & 0.009 & -11.05 & -12.68 & -34.89 & 0.778 \\
\hline & $\mathrm{C}$ & 0.629 & 0.127 & 0.244 & 0.065 & 0.015 & -7.28 & -9.44 & -25.97 & 0.433 \\
\hline \multirow[t]{3}{*}{4} & A & 0.868 & 0.056 & 0.076 & 0.081 & 0.005 & -8.51 & -10.71 & -28.85 & 0.740 \\
\hline & B & 0.459 & 0.296 & 0.245 & 0.047 & 0.019 & -5.46 & -8.21 & -20.61 & 0.450 \\
\hline & $\mathrm{C}$ & 0.868 & 0.056 & 0.076 & 0.081 & 0.005 & -8.51 & -10.70 & -28.81 & 0.740 \\
\hline \multirow[t]{4}{*}{5} & A & 0.901 & 0.041 & 0.058 & 0.085 & 0.003 & -7.43 & -9.59 & -25.68 & 0.816 \\
\hline & B & 0.097 & 0.758 & 0.145 & 0.028 & 0.023 & -1.93 & -5.30 & -10.38 & 0.517 \\
\hline & $\mathrm{C}$ & 0.901 & 0.041 & 0.058 & 0.085 & 0.003 & -7.51 & -9.87 & -25.79 & 0.816 \\
\hline & D & 0.901 & 0.041 & 0.058 & 0.085 & 0.003 & -7.59 & -9.89 & -25.94 & 0.816 \\
\hline 6 & A & -3.920 & 1.050 & 3.869 & - & 0.102 & 27.21 & 17.92 & 55.95 & -0.957 \\
\hline \multirow[t]{2}{*}{7} & A & 0.694 & 0.011 & 0.296 & 0.087 & 0.013 & -0.18 & -2.23 & -6.06 & 0.425 \\
\hline & B & -1.473 & 1.058 & 1.415 & - & 0.063 & 24.73 & 14.13 & 48.59 & -0.322 \\
\hline \multirow[t]{3}{*}{8} & A & 0.861 & 0.012 & 0.127 & 0.089 & 0.005 & -2.16 & -4.27 & -11.80 & 0.689 \\
\hline & B & -0.921 & 1.421 & 0.501 & - & 0.043 & 20.42 & 10.03 & 38.26 & 0.064 \\
\hline & $\mathrm{C}$ & 0.861 & 0.012 & 0.127 & 0.089 & 0.005 & -2.24 & -4.31 & -11.92 & 0.689 \\
\hline \multirow[t]{2}{*}{9} & A & 0.572 & 0.079 & 0.350 & - & 0.010 & -5.27 & -7.52 & -21.84 & 0.735 \\
\hline & B & 0.372 & 0.257 & 0.371 & - & 0.015 & -16.94 & -17.55 & -50.48 & 0.676 \\
\hline \multirow[t]{4}{*}{10} & A & 0.774 & 0.010 & 0.217 & 0.068 & 0.010 & 0.93 & -1.71 & -2.39 & 0.600 \\
\hline & B & -0.105 & 0.418 & 0.687 & - & 0.040 & 17.76 & 11.54 & 38.48 & 0.025 \\
\hline & $\mathrm{C}$ & 0.774 & 0.010 & 0.217 & 0.068 & 0.010 & 0.92 & -1.71 & -2.37 & 0.600 \\
\hline & D & -0.105 & 0.418 & 0.687 & - & 0.040 & 17.70 & 11.54 & 38.49 & 0.025 \\
\hline \multirow[t]{4}{*}{11} & A & 0.884 & 0.062 & 0.054 & 0.069 & 0.006 & -11.13 & -12.71 & -36.23 & 0.794 \\
\hline & B & 0.624 & 0.187 & 0.188 & 0.044 & 0.018 & -3.55 & -6.62 & -16.87 & 0.407 \\
\hline & $\mathrm{C}$ & 0.624 & 0.187 & 0.188 & 0.044 & 0.018 & -3.72 & -6.68 & -17.06 & 0.407 \\
\hline & $\mathrm{D}$ & 0.884 & 0.062 & 0.054 & 0.069 & 0.006 & -11.05 & -12.68 & -36.14 & 0.794 \\
\hline 12 & A & -0.227 & 0.296 & 0.931 & - & 0.045 & 1.24 & -2.03 & -4.55 & -0.076 \\
\hline 13 & A & -0.191 & 0.051 & 1.140 & - & 0.055 & 5.40 & - & - & -0.446 \\
\hline \multirow[t]{5}{*}{14} & A & -1.065 & 0.254 & 1.810 & - & 0.052 & 0.39 & 0.26 & 5.07 & -0.262 \\
\hline & B & -0.985 & 1.207 & 0.774 & - & 0.053 & 5.85 & -0.03 & 6.72 & -0.071 \\
\hline & C & -0.985 & 1.207 & 0.774 & - & 0.053 & 5.82 & -0.04 & 6.72 & -0.071 \\
\hline & D & -0.985 & 1.207 & 0.774 & - & 0.053 & 5.75 & -0.03 & 6.72 & -0.071 \\
\hline & E & -0.985 & 1.207 & 0.774 & - & 0.053 & 5.77 & -0.03 & 6.73 & -0.071 \\
\hline
\end{tabular}

Ring notation shown in Fig. 1

rings is considered. That is why we performed such a comparison, giving the correlation coefficients matrix in Tables 3 and 4 and some graphical representations in Fig. 2. The trends as concerns the changes in general are the same for all aromaticity indices, although some relations are rather far from pure linear regression. This most probably results from the fact that several different types of rings were taken into account in our studies. However, we did not expect linear regressions for all indices since we believe, in agreement with what was postulated earlier, that aromaticity is a collective phenomenon, and different criteria do not always give the same result [60-63]. Hence a variety of criteria should be used in the discussion of this term.

Final recommendations

In the last chapter of our article, we would like to give some recommendations regarding the use of EL index of aromaticity. We list these recommendations below: 
Fig. 2 Graphical

representations of interrelation between selected aromaticity indices. Always the maximum possible number of rings was taken, into account. However, in the case of PDI only six-

membered rings were

considered due to restrictions

resulting from definition of that aromaticity index
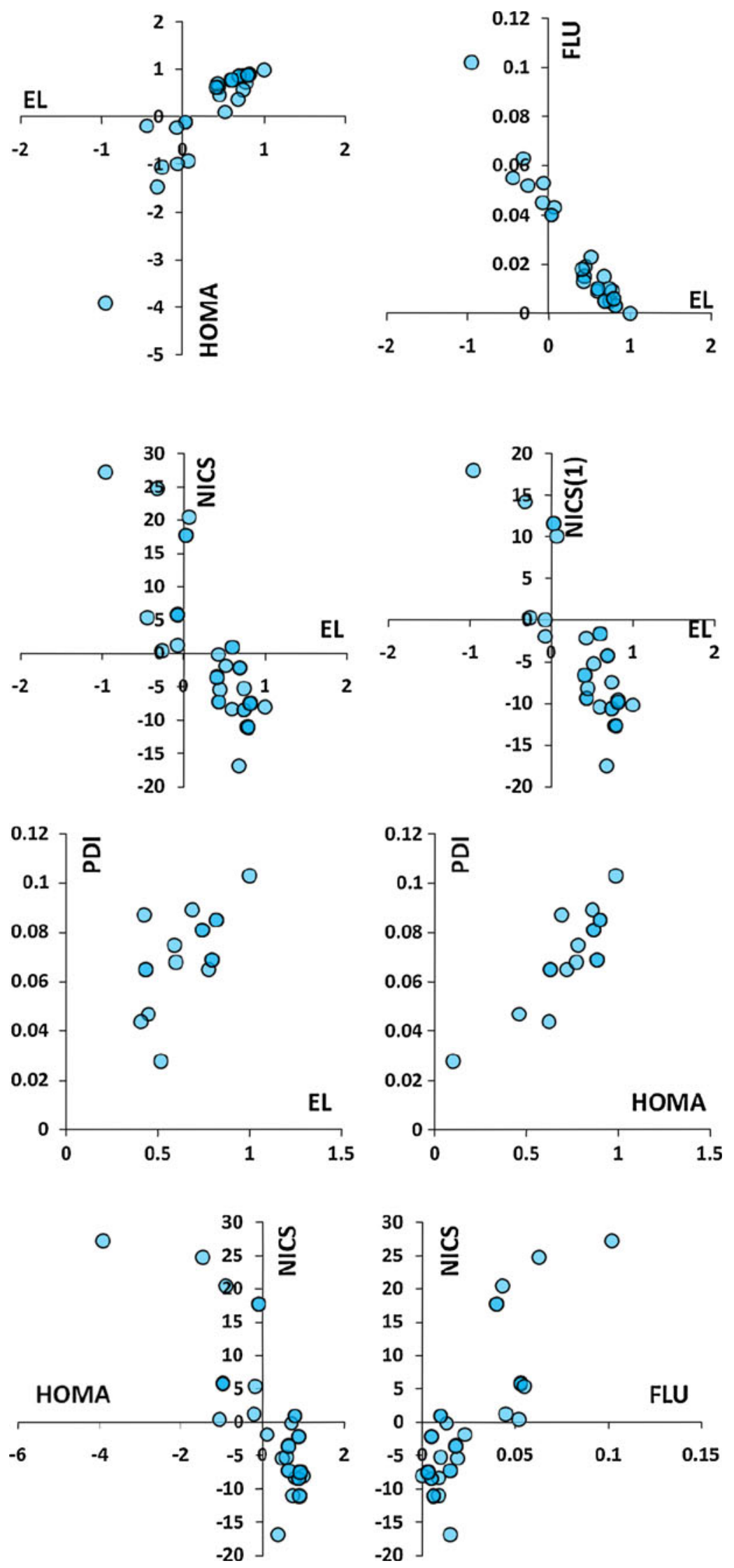
Table 3 Correlation coefficients estimated for linear regressions between values of HOMA, FLU, and three NICS indices

\begin{tabular}{lrrrrr}
\hline & HOMA & FLU & NICS & NICS $(1)$ & NICS $(1)_{\text {zz }}$ \\
\hline HOMA & 1.000 & & & \\
FLU & -0.977 & 1.000 & & & \\
NICS & -0.793 & 0.823 & 1.000 & & \\
NICS $(1)$ & -0.776 & 0.810 & 0.992 & 1.000 & 1.000 \\
NICS $(1)_{\text {zz }}$ & -0.786 & 0.823 & 0.995 & 0.998 & -0.840 \\
EL & 0.937 & -0.980 & -0.831 & -0.832 & 1.000
\end{tabular}

All rings in molecules from Fig. 1 were taken into account, with the only exception for the ring in system 13, which is not planar and for which the use of NICS(1) and NICS(1) $)_{\mathrm{zz}}$ is not justified

Table 4 Correlation coefficients estimated for linear regressions between values of HOMA, FLU, PDI, and three NICS indices

\begin{tabular}{|c|c|c|c|c|c|c|c|}
\hline & HOMA & FLU & PDI & NICS & NICS(1) & $\operatorname{NICS}(1)_{\mathrm{zz}}$ & EL \\
\hline HOMA & 1.000 & & & & & & \\
\hline FLU & -0.923 & 1.000 & & & & & \\
\hline PDI & 0.854 & -0.894 & 1.000 & & & & \\
\hline NICS & -0.348 & 0.386 & -0.182 & 1.000 & & & \\
\hline NICS(1) & -0.288 & 0.331 & -0.113 & 0.995 & 1.000 & & \\
\hline $\operatorname{NICS}(1)_{\mathrm{zz}}$ & -0.350 & 0.375 & -0.179 & 0.998 & 0.994 & 1.000 & \\
\hline EL & 0.700 & -0.886 & 0.660 & -0.491 & -0.460 & -0.474 & 1.000 \\
\hline
\end{tabular}

All six-membered rings in molecules from Fig. 1 were taken into account

1. The $c$ constant can be easily estimated for any level of calculations using the equation (6):

$c=\frac{n}{\sum_{i=1}^{n}\left|\varepsilon_{i}-\varepsilon_{\mathrm{ref}}\right|}$

where $\varepsilon_{i}$ denotes the ellipticities of single and inner double bonds in the reference system, which is the molecule of cyclohexa-1,3,5-triene with double and single bond lengths corresponding to the lengths of the inner double bond and the single bond in (3E)-hexa-1,3,5-triene.

2. We give here the values of $c$ constant estimated with the use of conditions given in point 1 for B3LYP/augcc-pVTZ and B3LYP/6-311++G(d,p) levels of calculations.

for B3LYP/aug-cc-pVTZ $c=10.6428$

for B3LYP/6-311++G(d,p) $c=10.0588$

One may use these values in the case of experimental charge density data or — when it is recommended —other data obtained at any level of calculations, since the error made using the value of $c$ estimated with the use of arbitrarily chosen level of calculations in such cases will be kept constant, making the comparative studies possible.

3. In general the level of calculations does not affect the final result of QTAIM calculations, as it was demonstrated for several methods and basis sets [64, 65]. Thus, the same could be expected in the case of EL use. However, the use of large basis sets with diffuse and polarization functions, wherever possible, is always recommended, since small basis sets may sometimes give artificial results [66].

4. As each aromaticity index, also EL has its limitations. First of all it depends on reference system. Therefore, similarly as in the case of other indices depending on reference data, the use of EL may be limited in specific cases, for instance, when the delocalization in the system under consideration does not result directly from its ground-state properties (e.g., in case of some transition or excited states). The use of EL is also limited to these systems for which the definition of the reference systems is possible at all. For example, the analysis of aromaticity in all-metal clusters with the use of EL can be rather difficult. Other significant limitation of EL results from the fact that it is based on the curvature of electron density in directions perpendicular to the bond path. It is related to the difference between two corresponding eigenvalues. The larger is the value of this difference in respect to the absolute values of both eigenvectors, the less reliable are indications of EL. In other words, EL should perform very well for covalent bonds, but its reliability will decrease with the increasing ionic character of bonds.

5. Similarly as HOMA and FLU, the use of EL is not limited to the cyclic systems only. EL can be used for the analysis of delocalization in any sequence of bonds, for which such analysis is justified. 


\section{Conclusions}

We demonstrate that ellipticity of the bond can be considered as the parameter useful for construction of a new index of aromaticity. We develop such a new measure of aromaticity - the EL index. Since EL can be estimated in standard QTAIM calculations on one-electron density function, it may serve as useful parameter not only in the case of theoretical approaches, but also for experimental data obtained from X-ray charge density studies. In this article, we check EL against several types of aromaticity indices. The indications are in general the same, which proves that EL can be considered as a new index of aromaticity.

Acknowledgments Calculations using the Gaussian09 set of codes were carried out in Wroclaw Center for Networking and Supercomputing (http://www.wcss.wroc.pl). Access to HPC machines and licensed software is gratefully acknowledged. This research was supported in part by PL-Grid Infrastructure.

Open Access This article is distributed under the terms of the Creative Commons Attribution Noncommercial License which permits any noncommercial use, distribution, and reproduction in any medium, provided the original author(s) and source are credited.

\section{References}

1. Schleyer PR (2001) Chem Rev 101:1115

2. Randic M (2003) Chem Rev 103:3449

3. Krygowski TM, Cyranski MK, Haflinger G, Katritzky AR (2000) Tetrahedron 56:1783

4. Soncini A, Steiner E, Fowler PW, Havenith RWA, Jenneskens LW (2003) Chem Eur J 9:2974

5. De Proft F, Fowler PW, Havenith RWA, Schleyer PvR, Van Lier G, Geerlings P (2004) Chem Eur J 10:940

6. Torrent-Sucarrat M, Luis JM, Sola M (2005) Chem Eur J 11:6024

7. Chen Z, Wannere CS, Corminboeuf C, Puchta R, Schleyer PR (2005) Chem Rev 105:3842

8. Stanger A (2006) Chem Eur J 12:2745

9. Stanger A (2006) J Org Chem 71:883

10. Hajgato B, Deleuze MS, Ohno K (2006) Chem Eur J 12:5757

11. Bultinck P, Fias S, Ponec R (2006) Chem Eur J 12:8813

12. Pascal RA Jr (2006) Chem Rev 106:4809

13. Balaban A (2011) Phys Chem Chem Phys 13:20649

14. Ulusoy IS, Nest M (2011) doi:10.1021/ja206193t

15. Matito E, Duran M, Sola M (2005) 122:0141091 erratum (2006) J Chem Phys 125:059901

16. Kruszewski J, Krygowski TM (1972) Tetrahedron Lett 13:3839

17. Krygowski TM (1993) J Inf Comput Sci 33:70

18. Schleyer PR, Maerker C, Dransfeld A, Jiao H, Hommes NJRE (1996) J Am Chem Soc 118:6317

19. Islas R, Heine $T$, Merino $G$ (2011) Acc Chem Res. doi: 10.1021/ar200117a

20. Poater J, Fradera X, Duran M, Sola M (2003) Chem Eur J 9:400

21. Bultinck P, Rafat M, Ponec R, Gheluwe B, Carbo-Dorca R, Popelier P (2006) J Phys Chem A 110:7642

22. Bultinck P, Ponec R, Van Damme S (2005) J Phys Org Chem 18:706
23. Matta CF, Hernández-Trujillo J (2005) J Phys Chem A 109:10798

24. Bader RFW (1990) In: Atoms in molecules. A quantum theory. Oxford University Press, New York

25. Hohenberg P, Kohn W (1964) Phys Rev B 136:864

26. Kohn W, Sham LJ (1965) Phys Rev A 140:1133

27. Frisch MJ, Trucks GW, Schlegel HB, Scuseria GE, Robb MA, Cheeseman JR, Scalmani G, Barone V, Mennucci B, Petersson GA, Nakatsuji H, Caricato M, Li X, Hratchian HP, Izmaylov AF, Bloino J, Zheng G, Sonnenberg JL, Hada M, Ehara M, Toyota K, Fukuda R, Hasegawa J, Ishida M, Nakajima T, Honda Y, Kitao O, Nakai H, Vreven T, Montgomery Jr JA, Peralta JE, Ogliaro F, Bearpark M, Heyd JJ, Brothers E, Kudin KN, Staroverov VN, Kobayashi R, Normand J, Raghavachari K, Rendell A, Burant JC, Iyengar SS, Tomasi J, Cossi M, Rega N, Millam JM, Klene M, Knox JE, Cross JB, Bakken V, Adamo C, Jaramillo J, Gomperts R, Stratmann RE, Yazyev O, Austin AJ, Cammi R, Pomelli C, Ochterski JW, Martin RL, Morokuma K, Zakrzewski VG, Voth GA, Salvador P, Dannenberg JJ, Dapprich S, Daniels AD, Farkas O, Foresman JB, Ortiz JV, Cioslowski J, Fox DJ (2009) In: Gaussian 09 (Revision A.02). Gaussian Inc, Wallingford

28. Becke AD (1993) J Chem Phys 98:5648

29. Lee C, Yang W, Parr RG (1988) Phys Rev B 37:785

30. Stephens PJ, Devlin FJ, Chabalowski CF, Frisch MJ (1994) J Phys Chem 98:11623

31. Miehlich B, Savin A, Stoll H, Preuss H (1989) Chem Phys Lett 157:200

32. Krishnan R, Binkley JS, Seeger R, Pople JA (1980) J Chem Phys 72:650

33. Bader RFW (1991) Chem Rev 91:893

34. Keith TA (2010) AIMAll program Version 10.12.11. http://aim. tkgristmill.com

35. Krygowski TM, Cyrański MK (1996) Tetrahedron 52:1713

36. Krygowski TM, Cyrański MK (1996) Tetrahedron 52:10255

37. Fradera X, Austen MA, Bader RFW (1999) J Phys Chem A 103:304

38. Fradera X, Poater J, Simon S, Duran M, Sola M (2002) Theor Chem Acc 108:214

39. Popelier PLA (2000) In: Atoms in molecules, an introduction. Prentice Hall, Harlow

40. O'Brien SE, Popelier PLA (1999) Can J Chem 77:28

41. Merino G, Vela A, Heine T (2005) Chem Rev 105:3812

42. Stalke D (2011) Chem Eur J 17:9264

43. Bader RFW (1998) J Phys Chem A 102:7314

44. Bader RFW (2009) J Phys Chem A 113:10391 (and references therein)

45. Howard ST, Krygowski TM (1997) Can J Chem 75:1174

46. Bader RWF, Matta CF (2004) Organometallics 23:6253

47. Vidal I, Melchor S, Alkorta I, Elguero J, Sundberg MR, Dobado JA (2006) Organometallics 25:5638

48. Palusiak M (2007) J Organomet Chem 692:3866

49. Strzelczyk W, Sobieszczyk P, Palusiak M (2009) Struct Chem 20:919

50. Dominikowska J, Palusiak M (2010) New J Chem 34:1855

51. Grabowski SJ (2011) Chem Rev 111:2597

52. Sobczyk L, Grabowski SJ, Krygowski TM (2005) Chem Rev 105:3513

53. Gonzalez L, Mo O, Yanez M, Elguero J (1996) J Mol Struct 371:1

54. Espinosa E, Souhassou M, Lachekar H, Lecomte C (1999) Acta Crystallogr B 55:563

55. Grabowski SJ (2000) J Phys Chem A 104:5551

56. Mo O, Yanez M, Pendas AM, Del Bene JE, Alkorta I, Elguero J (2007) Phys Chem Chem Phys 9:3970

57. Cremer D, Kraka E (1984) Angew Chem Int Ed Engl 23:627

58. Bankiewicz B, Palusiak M (2011) Comput Theor Chem 966:113 
59. Clar E (1972) In: The aromatic sextet. Wiley, New York

60. Katritzky AR, Barczynski P, Mussumura G, Pisano D, Szafran M (1989) J Am Chem Soc 111:7

61. Jug K, Koester A (1991) J Phys Org Chem 4:163

62. Krygowski TM, Ciesielski A, Bird CW, Kotschy A (1995) J Chem Inf Comput Sci 35:203
63. Cyrański MK, Krygowski TM, Katritzky AR, Schleyer PR (2002) J Org Chem 67:1333

64. Jabłoński M, Palusiak M (2010) J Phys Chem A 114:2240

65. Jabłoński M, Palusiak M (2010) J Phys Chem A 114:12498

66. Dominikowska J, Palusiak M (2011) J Comput Chem 32:1441 